\title{
Structural Parameter Identification of Articulated Arm Coordinate Measuring Machines
}

\author{
Guanbin Gao, Huaishan Zhang, Xing Wu, and Yu Guo \\ Faculty of Mechanical and Electrical Engineering, Kunming University of Science and Technology, Kunming, China \\ Correspondence should be addressed to Guanbin Gao; gbgao@163.com
}

Received 29 February 2016; Revised 14 June 2016; Accepted 4 September 2016

Academic Editor: Tomonari Furukawa

Copyright (C) 2016 Guanbin Gao et al. This is an open access article distributed under the Creative Commons Attribution License, which permits unrestricted use, distribution, and reproduction in any medium, provided the original work is properly cited.

\begin{abstract}
Precise structural parameter identification of a robotic articulated arm coordinate measuring machine (AACMM) is essential for improving its measuring accuracy, particularly in robotic applications. This paper presents a constructive parameter identification approach for robotic AACMMs. We first develop a mathematical kinematic model of the AACMM based on the DenavitHartenberg $(\mathrm{DH})$ approach established for robotic systems. This model is further calibrated and verified via the practical test data. Based on the difference between the calculated coordinates of the AACMM probe via the kinematic model and the given reference coordinates, a parameter identification approach is proposed to estimate the structural parameters in terms of the test data set. The Jacobian matrix is further analyzed to determine the solvability of the identification model. It shows that there are two coupling parameters, which can be removed in the regressor. Finally, a parameter identification algorithm taking the least-square solution of the identification model as the structural parameters by using the obtained poses data is suggested. Practical experiments based on a robotic AACMM test rig are carried out, and the results reveal the effectiveness and robustness of the proposed identification approach.
\end{abstract}

\section{Introduction}

The coordinate measuring machine (CMM) is a universal measuring instrument which can transform various geometric measurements into coordinate measurements [1]. This instrument has been widely used in the calibration and modeling of robotic systems. In particular, the articulated arm CMM (AACMM) is a new type of robot-like CMM with multiple degrees of freedom (DOF), which generally consists of a series of linkages connected by joints in series $[2,3]$. The AACMM obtains the angles of joints by means of the angle encoder installed on the rotary joints. And the angles can be transformed into the three coordinates $(x, y, z)$ through the kinematic model. The AACMM possesses some specific and essential characteristics and advantages, for example, simple mechanical structure, small size, light weight, large measurement range, and flexible measurement in field [4].

However, the measuring accuracy of the AACMM is much lower than that of the orthogonal CMM $[5,6]$, which may greatly limit its applications. A potential strategy to improve the measuring accuracy of the AACMM is to choose high precision hardware components and to apply high requirements for manufacturing and assembly [3, 7]. However, the cost of the measuring machine increases dramatically by using more precise components. Moreover, in some specific applications, increasing the precision of individual components may not be able to ensure overall increased measuring precision and accuracy. In particular, small errors in some kinematic parameters may accumulate and thus influence the measuring accuracy of the AACMM greatly.

Another essential and economic way to eliminate errors of the structural parameters and to improve the measuring accuracy is to identify the robot's structural parameters [8] by using appropriate parameter identification approaches. Generally, the structural parameter identification includes four steps [9]: (1) modeling: to establish a mathematical model describing the geometrical characteristics and kinematics of the robot; (2) measurement: to measure the coordinates of the end effector in the real world coordinate system; (3) identification: to identify the structural parameters of the obtained model by means of mathematical calculation of the data set; (4) compensation: to modify the parameters 
in the control system according to the identification results. These four steps are also applicable for the AACMM, and an improved identification method will be studied in this paper.

For the parameter identification of AACMM, Kovač and Frank [10] developed a new high precision device for the AACMM testing and calibration with the laser interferometer measurements along a line gauge beam. Santolaria et al. $[11,12]$ reported a method to calibrate an AACMM based on the Denavit-Hartenberg (DH) kinematic model parameters. These parameters are optimized by measuring a calibrated ball bar gauge located at different orientations and positions in the AACMM working space. Hamana et al. [13] presented a method, where the kinematic parameters of AACMM were calibrated using spherical center coordinates as the artifact. However, only part of the measuring space can be calibrated with the above methods. Thus, the AACMM cannot be calibrated by directly using these available results. In particular, the coupling relationships between the structural parameters and their effect on the measuring uncertainty of the AACMM were not considered in the above researches. Therefore, the robustness and efficiency of the identifications were affected due to those invalid calculations [14] of the redundant couplings.

In this paper, we propose an improved modeling and parameter identification method for AACMM robotic system. First, the kinematic model and structural identification matrix were established based on the $\mathrm{DH}$ method, and the coupling relationship between the structural parameters was obtained through further analysis of the structural identification matrix. Then the identification model of the AACMM was constructed, and a parameter estimation approach developed based on the LS method is proposed to identify the structural parameters. Practically collected data of the joint angles and coordinates of the probe are used to validate the model and identification approach. The redundancy embedded in the parameter matrix is further analyzed and eliminated to address the coupling effects and identifiability. Finally, practical experiments are conducted to verify the efficiency of the proposed identification method.

The advantages and the distinctive features of this proposed identification method in comparison to some other identification methods for AACMM (e.g., $[12,15,16])$ are as follows:

(1) We do not need precise initial parameters, and even we do not need initial parameters (we can assign the initial parameters arbitrarily as long as they are not too exaggerated) in the identification. The identified values of the structural parameters can be solved through (18). However, in some available results, for example, $[12,15,17]$, the initial identification parameters should be appropriately selected to achieve good identification results because an iteration calculation method is adopted.

(2) In this paper, we conducted the coupling analysis such that those linearly dependant parameters are detected and removed from the parameters to be identified. Consequently, the calculation costs and the identification efficacy can be significantly improved.

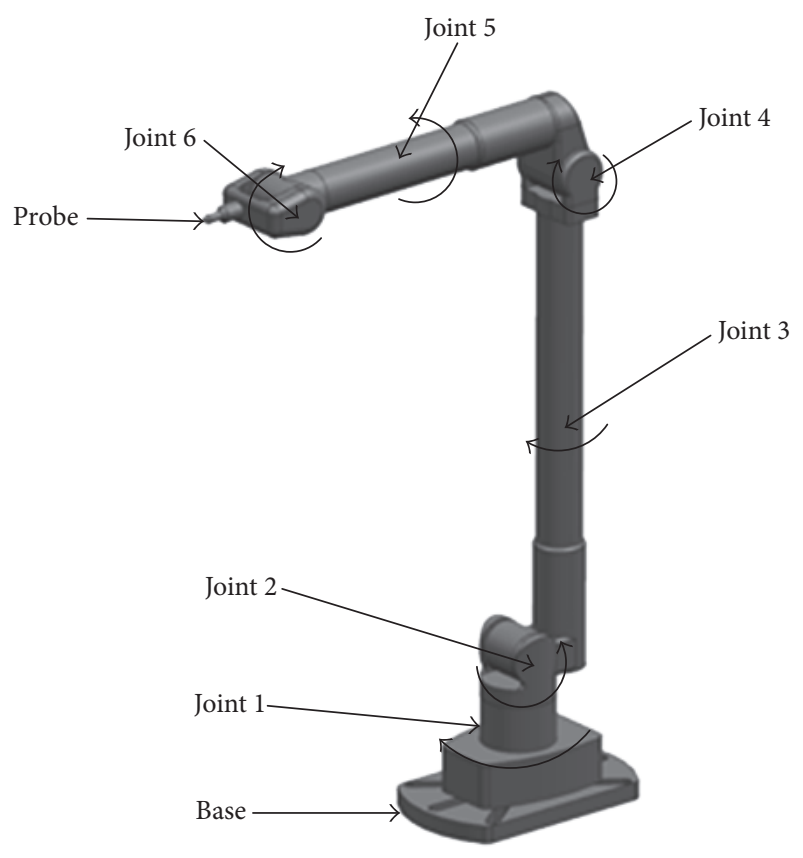

FIgURE 1: The structure of the AACMM.

In fact, in our case study, only one time iteration calculation can provide fairly good results.

(3) The time consumed by the proposed identification calculation is relatively shorter than the widely used iteration identification methods such as PSO, GA, and LS; that is, we can get the results just after one time iteration calculation.

The paper is organized as follows. Section 2 presents the kinematic modeling and validation; the parameter identification and the analysis are introduced in Section 3; and experimental results are given in Section 4 . Section 5 provides conclusions.

\section{Kinematic Modeling and Verification}

2.1. Kinematic Modeling. As shown in Figure 1, the structure of the AACMM is similar to an articulated robot. Therefore, the AACMM model can be established by using available modeling methods developed for robotics. For the modeling of robotic kinematics, the most influential method is the Denavit-Hartenberg model (DH model) which has been widely used due to its clear physical meaning [18]. A $4 \times$ 4 homogeneous transform matrix is used to represent the spatial relations of adjacent joints coordinate systems [19]. Because all the adjacent joints of the AACMM are perpendicular [20], there is no nominally parallel problem, and we can use the DH method to establish the kinematic model.

In this paper, a 6-DOF AACMM is studied. The schematic structure of this system is shown in Figure 1. For this 6-DOF AACMM, the coordinate values of the probe in the reference coordinate system can be derived through 7 successive transformations. 


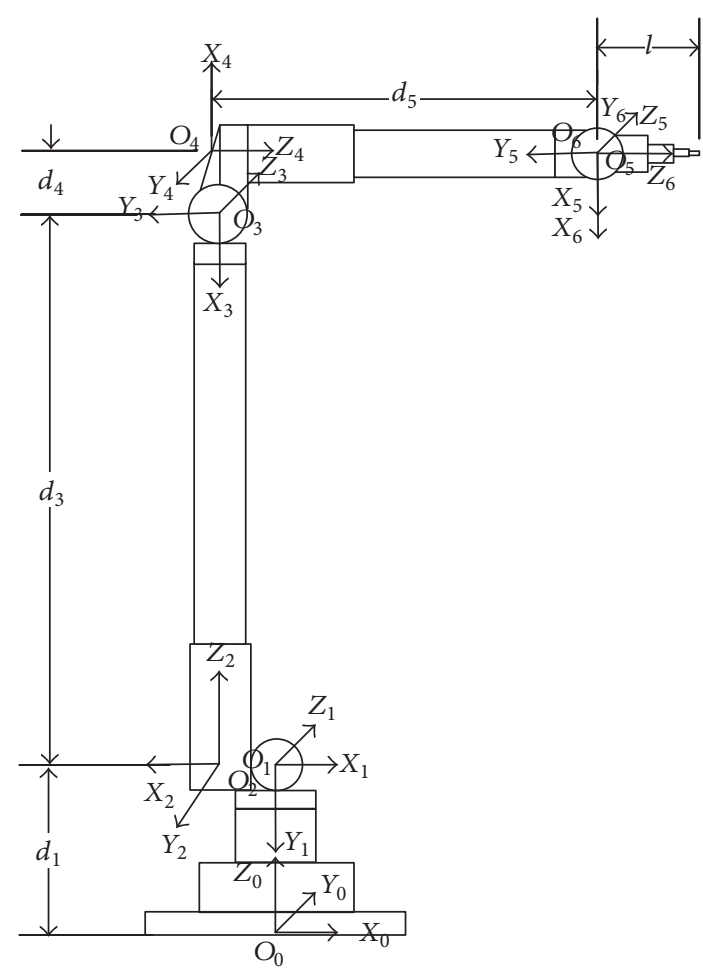

FIGURE 2: Coordinate systems of the AACMM.

TABLE 1: The estimated structural parameters of the AACMM.

\begin{tabular}{lcccc}
\hline Linkage number $i$ & $a_{i}[\mathrm{~mm}]$ & $d_{i}[\mathrm{~mm}]$ & $\Delta \theta_{i}\left[{ }^{\circ}\right]$ & $\alpha_{i}\left[^{\circ}\right]$ \\
\hline 1 & 0 & 376 & 0 & -90 \\
2 & 62 & 0 & 0 & -90 \\
3 & 0 & 751 & 0 & -90 \\
4 & 62 & 0 & 0 & -90 \\
5 & 0 & 500 & 0 & -90 \\
6 & 0 & 15 & 0 & 90 \\
\hline
\end{tabular}

$l=98 \mathrm{~mm}$.

According to the DH method, there are four groups of structural parameters in the AACMM, for example, linkage length $d_{i}$, joint length $a_{i}$, torsion angle $\alpha_{i}$, and joint angle $\theta_{i}$.

The coordinates of the AACMM were established followed by the DH method, as shown in Figure 2. Some of the four groups of structural parameters were achieved via direct measurement and some of them can only be determined by using estimation method because they cannot be measured directly, for example, $\alpha_{i}$, as shown in Table 1 . The measurement datum of the structural parameters is all axes of the joints and linkages, which are virtual lines only, and the positions of them are not accurate. Therefore, the structural parameters are not accurate as they will result in significant movement or measurement uncertainty of the AACMM.

According to the principle of homogeneous transformation, the transform from the coordinate system $\left\{O_{i} X_{i} Y_{i} Z_{i}\right\}$ to $\left\{O_{i-1} X_{i-1} Y_{i-1} Z_{i-1}\right\}$ is equivalent to a process in which $\left\{O_{i-1} X_{i-1} Y_{i-1} Z_{i-1}\right\}$ conducts rotation and translation and

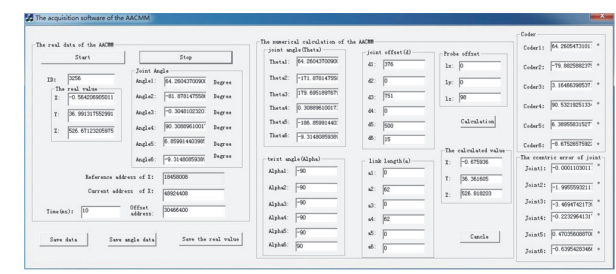

FIgURE 3: The interface of the acquisition software of the AACMM.

then completely coincides with $\left\{O_{i} X_{i} Y_{i} Z_{i}\right\}$. The whole transform procedure can be expressed mathematically as

$$
\begin{aligned}
T_{i-1, i} & =\operatorname{Rot}\left(z_{i-1}, \theta_{i}\right) \operatorname{Trans}\left(0,0, d_{i}\right) \operatorname{Trans}\left(a_{i}, 0,0\right) \\
\cdot & \operatorname{Rot}\left(x_{i}, \alpha_{i}\right) \\
& =\left[\begin{array}{cccc}
\cos \theta_{i} & -\sin \theta_{i} \cos \alpha_{i} & \sin \theta_{i} \sin \alpha_{i} & a_{i} \cos \theta_{i} \\
\sin \theta_{i} & \cos \theta_{i} \cos \alpha_{i} & -\cos \theta_{i} \sin \alpha_{i} & a_{i} \sin \theta_{i} \\
0 & \sin \alpha_{i} & \cos \alpha_{i} & d_{i} \\
0 & 0 & 0 & 1
\end{array}\right] .
\end{aligned}
$$

The probe coordinates in the reference coordinate system $\left\{\mathrm{O}_{0} X_{0} Y_{0} Z_{0}\right\}$ can be expressed as follows:

$$
\begin{aligned}
& {\left[\begin{array}{l}
x \\
y \\
z \\
1
\end{array}\right]=T_{0,1} \cdot T_{1,2} \cdot T_{2,3} \cdot T_{3,4} \cdot T_{4,5} \cdot T_{5,6} \cdot\left[\begin{array}{l}
0 \\
0 \\
l \\
1
\end{array}\right]} \\
& =\prod_{i=1}^{6}\left[\begin{array}{cccc}
\cos \theta_{i} & -\sin \theta_{i} \cos \alpha_{i} & \sin \theta_{i} \sin \alpha_{i} & a_{i} \cos \theta_{i} \\
\sin \theta_{i} & \cos \theta_{i} \cos \alpha_{i} & -\cos \theta_{i} \sin \alpha_{i} & a_{i} \sin \theta_{i} \\
0 & \sin \alpha_{i} & \cos \alpha_{i} & d_{i} \\
0 & 0 & 0 & 1
\end{array}\right]
\end{aligned}
$$

$$
\cdot\left[\begin{array}{l}
0 \\
0 \\
l \\
1
\end{array}\right] \text {. }
$$

There is one group of parameters which are the variables in (2). It is the joint angle $\theta_{i}$, the value of which can be acquired by the joint angular sensor.

2.2. Verification of the Kinematic Model. To validate the proposed kinematic model (2), we collect realistic operation data via sensors, for example, joint angle of the AACMM, which are then used to calculate the coordinate system. For this purpose, a software according to the kinematic model (2) was developed to acquire the joint angles, which can also be used to verify the kinematic model of the AACMM. The interface of the built software with $\mathrm{C}++$ is shown in Figure 3. As we find all the structural parameters can be imported into the software through the interface. Then with the joint angles 
acquired from the AACMM and the structural parameters, the coordinates of the AACMM probe can be calculated by the software.

By comparing the coordinates of the probe calculated by the software and the reference coordinates (which can be treated as the true values of the coordinates of the probe), we found that the calculated coordinates of the probe are very close to the reference coordinates. The differences between the two group coordinates are always less than $1.5 \mathrm{~mm}$, which means that the movement uncertainty of the AACMM is about $1.5 \mathrm{~mm}$. In this sense, the kinematic model (2) of the AACMM is correct because a wrong kinematic model will lead to uncontrollable movement uncertainty. However, it should be noted that although such movement uncertainty is acceptable for industrial robots, it is not applicable for AACMM as this is a much more precision instrument. Thus further analysis will be conducted in the following Section 3 to identify the exact structural parameters by introducing a discrete-time parameter identification method.

\section{Identification for AACMM Uncertain Parameters}

3.1. Modeling Error Analysis. It is known that the accuracy of the AACMM is affected greatly by the manufacture, assembly, and component selection [21, 22], which can all result in inconsistence between the structural parameters in the kinematic model and the actual ones. However, the proposed identification method in this paper can achieve high accuracy for AACMM modeling without increasing the cost to construct the parts and buy extra sensors. This section will further address the improvement of accuracy by analyzing the error dynamics and then introducing an associated identification scheme.

From (2), we know that the uncertainty of the AACMM is affected by the error of structural parameters including the linkage length error $\Delta d_{i}$, the torsion angle error $\Delta \alpha_{i}$, the joint length error $\Delta a_{i}$, the probe length error $\Delta l$, and the joint angle deviation $\Delta \theta_{i}$ at the zero position [23]. Thus the effects of these uncertainties should be identified and then compensated. For this purpose, (2) can be rewritten as a function group of the structural parameters, which is shown in

$$
\begin{aligned}
x= & f_{x}\left(\theta_{1}, \theta_{1}, \theta_{1}, \theta_{1}, \theta_{1}, \theta_{1}, \alpha_{1}, \alpha_{2}, \alpha_{3}, \alpha_{4}, \alpha_{5}, \alpha_{6}, d_{1}, d_{2}, d_{3}, d_{4},\right. \\
& \left.d_{5}, d_{6}, a_{1}, a_{2}, a_{3}, a_{4}, a_{5}, a_{6}, l\right) \\
y= & f_{x}\left(\theta_{1}, \theta_{1}, \theta_{1}, \theta_{1}, \theta_{1}, \theta_{1}, \alpha_{1}, \alpha_{2}, \alpha_{3}, \alpha_{4}, \alpha_{5}, \alpha_{6}, d_{1}, d_{2}, d_{3}, d_{4},\right. \\
& \left.d_{5}, d_{6}, a_{1}, a_{2}, a_{3}, a_{4}, a_{5}, a_{6}, l\right) \\
z= & f_{x}\left(\theta_{1}, \theta_{1}, \theta_{1}, \theta_{1}, \theta_{1}, \theta_{1}, \alpha_{1}, \alpha_{2}, \alpha_{3}, \alpha_{4}, \alpha_{5}, \alpha_{6}, d_{1}, d_{2}, d_{3}, d_{4},\right. \\
& \left.d_{5}, d_{6}, a_{1}, a_{2}, a_{3}, a_{4}, a_{5}, a_{6}, l\right) .
\end{aligned}
$$

To show the effect of uncertainties, we calculate the differential of (3) as

$$
\begin{aligned}
d x= & \sum_{i=1}^{6} \frac{\partial f_{x}}{\partial \theta_{i}} d \theta_{i}+\sum_{i=1}^{6} \frac{\partial f_{x}}{\partial \alpha_{i}} d \alpha_{i}+\sum_{i=1}^{6} \frac{\partial f_{x}}{\partial d_{i}} d d_{i}+\sum_{i=1}^{6} \frac{\partial f_{x}}{\partial a_{i}} d a_{i} \\
& +\frac{\partial f_{x}}{\partial l} d l \\
d y= & \sum_{i=1}^{6} \frac{\partial f_{y}}{\partial \theta_{i}} d \theta_{i}+\sum_{i=1}^{6} \frac{\partial f y}{\partial \alpha_{i}} d \alpha_{i}+\sum_{i=1}^{6} \frac{\partial f_{y}}{\partial d_{i}} d d_{i}+\sum_{i=1}^{6} \frac{\partial f_{y}}{\partial a_{i}} d a_{i} \\
& +\frac{\partial f_{y}}{\partial l} d l \\
d z= & \sum_{i=1}^{6} \frac{\partial f_{z}}{\partial \theta_{i}} d \theta_{i}+\sum_{i=1}^{6} \frac{\partial f_{z}}{\partial \alpha_{i}} d \alpha_{i}+\sum_{i=1}^{6} \frac{\partial f_{z}}{\partial d_{i}} d d_{i}+\sum_{i=1}^{6} \frac{\partial f_{z}}{\partial a_{i}} d a_{i} \\
& +\frac{\partial f_{z}}{\partial l} d l .
\end{aligned}
$$

Thus (4) can be rewritten in a compact form as given in

$$
\begin{aligned}
& {\left[\begin{array}{l}
d x \\
d y \\
d z
\end{array}\right]=\left[\begin{array}{l}
\frac{\partial f_{x}}{\partial \theta_{1}} \cdots \frac{\partial f_{x}}{\partial \theta_{6}} \frac{\partial f_{x}}{\partial \alpha_{1}} \cdots \frac{\partial f_{x}}{\partial \alpha_{6}} \frac{\partial f_{x}}{\partial d_{1}} \cdots \frac{\partial f_{x}}{\partial d_{6}} \frac{\partial f_{x}}{\partial a_{1}} \cdots \frac{\partial f_{x}}{\partial a_{6}} \frac{\partial f_{x}}{\partial l} \\
\frac{\partial f_{y}}{\partial \theta_{1}} \cdots \frac{\partial f_{y}}{\partial \theta_{6}} \frac{\partial f_{y}}{\partial \alpha_{1}} \cdots \frac{\partial f_{y}}{\partial \alpha_{6}} \frac{\partial f_{y}}{\partial d_{1}} \cdots \frac{\partial f_{y}}{\partial d_{6}} \frac{\partial f_{y}}{\partial a_{1}} \cdots \frac{\partial f_{y}}{\partial a_{6}} \frac{\partial f_{y}}{\partial l} \\
\frac{\partial f_{z}}{\partial \theta_{1}} \cdots \frac{\partial f_{z}}{\partial \theta_{6}} \frac{\partial f_{z}}{\partial \alpha_{1}} \cdots \frac{\partial f_{z}}{\partial \alpha_{6}} \frac{\partial f_{z}}{\partial d_{1}} \cdots \frac{\partial f_{z}}{\partial d_{6}} \frac{\partial f_{z}}{\partial a_{1}} \cdots \frac{\partial f_{z}}{\partial a_{6}} \frac{\partial f_{z}}{\partial l}
\end{array}\right]} \\
& \times\left[\begin{array}{lllllllllllll}
d \theta_{1} & \cdots & d \theta_{6} & d \alpha_{1} & \cdots & d \alpha_{6} & d d_{1} & \cdots & d d_{6} & d a_{1} & \cdots & d a_{6} & d l
\end{array}\right]^{T} .
\end{aligned}
$$

It is shown that (5) is in a linear form with perturbing modeling uncertainties. Since we consider sufficiently small sampling interval in the discrete-time implementation, the structural parameters of the AACMM are relatively small; that is, $\Delta \theta_{i}=d \theta_{i}$ and $d x=\Delta x$. Then (5) can be rewritten as

$$
\left[\begin{array}{l}
\Delta x \\
\Delta y \\
\Delta z
\end{array}\right]=\mathbf{J} \times\left[\begin{array}{lllllllllllllll}
\Delta \theta_{1} & \cdots & \Delta \theta_{6} & \Delta \alpha_{1} & \cdots & \Delta \alpha_{6} & \cdots & \Delta d_{1} & \cdots & \Delta d_{6} & \cdots & \Delta a_{1} & \cdots & \Delta a_{6} & d l
\end{array}\right]^{T}
$$


We denote

J

$$
=\left[\begin{array}{l}
\frac{\partial f_{x}}{\partial \theta_{1}} \cdots \frac{\partial f_{x}}{\partial \theta_{6}} \frac{\partial f_{x}}{\partial \alpha_{1}} \cdots \frac{\partial f_{x}}{\partial \alpha_{6}} \frac{\partial f_{x}}{\partial d_{1}} \cdots \frac{\partial f_{x}}{\partial d_{6}} \frac{\partial f_{x}}{\partial a_{1}} \cdots \frac{\partial f_{x}}{\partial a_{6}} \frac{\partial f_{x}}{\partial l} \\
\frac{\partial f_{y}}{\partial \theta_{1}} \cdots \frac{\partial f_{y}}{\partial \theta_{6}} \frac{\partial f_{y}}{\partial \alpha_{1}} \cdots \frac{\partial f_{y}}{\partial \alpha_{6}} \frac{\partial f_{y}}{\partial d_{1}} \cdots \frac{\partial f_{y}}{\partial d_{6}} \frac{\partial f_{y}}{\partial a_{1}} \cdots \frac{\partial f_{y}}{\partial a_{6}} \frac{\partial f_{y}}{\partial l} \\
\frac{\partial f_{z}}{\partial \theta_{1}} \cdots \frac{\partial f_{z}}{\partial \theta_{6}} \frac{\partial f_{z}}{\partial \alpha_{1}} \cdots \frac{\partial f_{z}}{\partial \alpha_{6}} \frac{\partial f_{z}}{\partial d_{1}} \cdots \frac{\partial f_{z}}{\partial d_{6}} \frac{\partial f_{z}}{\partial a_{1}} \cdots \frac{\partial f_{z}}{\partial a_{6}} \frac{\partial f_{z}}{\partial l}
\end{array}\right]
$$

$$
\Delta \mathbf{X}=\left[\begin{array}{llllllllllllll}
\Delta \theta_{1} & \cdots & \Delta \theta_{6} & \Delta \alpha_{1} & \cdots & \Delta \alpha_{6} & \Delta d_{1} & \cdots & \Delta d_{6} & \cdots & \Delta a_{1} & \cdots & \Delta a_{6} & d l
\end{array}\right]^{T}
$$

as the Jacobian matrix,

$$
\Delta \mathbf{P}=\left[\begin{array}{c}
\Delta x \\
\Delta y \\
\Delta z
\end{array}\right]
$$

as the difference between the coordinates of the probe calculated by the kinematic model and the measured reference coordinates, and as the uncertainties perturbing the structure parameters. Then (6) can also be simplified as

$$
\Delta \mathbf{P}=\mathbf{J} \cdot \Delta \mathbf{X},
$$

where $\Delta \mathbf{X}=\mathbf{X}(k)-\mathbf{X}(k-1)$ denotes the difference of the regressor within each iteration step $[k-1, k]$.

Equation (10) is in a strictly linearly parameterized form within each iteration step, and thus it can be used for the purpose of structure parameter identification to estimate $\Delta \mathbf{X}$.

3.2. Identification of Structural Parameters. From (6), we know that (10) can be used to solve the error of structural parameters $\Delta X$. It should be noted that there are 25 unknown parameters in (10), for example, $\Delta \theta_{i}, \Delta \alpha_{i}, \Delta d_{i}, \Delta a_{i}, i=$ $1 \cdots 6$, and $\Delta l$. However, there are only 3 measurements (e.g., $\Delta x, \Delta y, \Delta z)$. Therefore, we need to collect at least 9 groups of reference coordinates and poses (joint angles) in various operation regimes to creates $9 \times 3=27$ equations, which are then used to identify these 25 unknown parameters. To get more robust results, we need even more than 9 groups of reference coordinates and joint angles. Thus, for $n \geq 9$, we can write augmented (10) as

$$
\left[\begin{array}{c}
\Delta \mathbf{P}_{1} \\
\Delta \mathbf{P}_{2} \\
\vdots \\
\Delta \mathbf{P}_{n}
\end{array}\right]=\left[\begin{array}{c}
\mathbf{J}_{1} \\
\mathbf{J}_{2} \\
\vdots \\
\mathbf{J}_{n}
\end{array}\right] \cdot \Delta \mathbf{X}, \quad n>9
$$

It is clear that the uncertain structural parameters $\Delta \mathbf{X}$ can be calculated based on (11) when the regressor or matrix is nonsingular. This is the well-known persistent excitation condition and can be fulfilled in our case study by operating the robotic in extensive scenarios. To solve $\Delta \mathbf{X}$ in (11) conveniently, (11) can be further rewritten in a more compact form

$$
\Delta \mathbf{X}=\left(\overline{\mathbf{J}}^{T} \cdot \overline{\mathbf{J}}\right)^{-1} \overline{\mathbf{J}} \Delta \overline{\mathbf{P}}
$$

where $\Delta \overline{\mathbf{P}}=\left[\Delta \mathbf{P}_{1}, \Delta \mathbf{P}_{2}, \ldots, \Delta \mathbf{P}_{n}\right]^{T}$ and $\overline{\mathbf{J}}=\left[\mathbf{J}_{1}, \mathbf{J}_{2}, \ldots, \mathbf{J}_{n}\right]^{T}$ denote the augmented regressor and measurement matrices.
3.3. Analysis of Couplings of the Structural Parameters. In (12), if the Jacobian matrix $\overline{\mathbf{J}}$ is not a column full rank matrix, that is, there are linear correlation structural parameters, we cannot calculate its solution directly. Therefore, we should check if there are linearly correlated structural parameters in $\overline{\mathbf{J}}$. Based on the matrix theory, the linearly related row can be found by applying singular value decomposition elementary row transformation of $\overline{\mathbf{J}}^{T} \cdot \overline{\mathbf{J}}$ via the orthogonal matrix decomposition $[24,25]$. Premultiplying $\overline{\mathbf{J}}^{T}$ on both sides of (12), we can obtain

$$
\left[\overline{\mathbf{J}}^{T} \overline{\mathbf{J}}\right] \Delta \mathbf{X}=\overline{\mathbf{J}}^{T} \Delta \overline{\mathbf{P}}
$$

We denote $\mathbf{H}=\left[\overline{\mathbf{J}}^{T} \overline{\mathbf{J}}\right]$; then by using the matrix singular value decomposition, we have

$$
\mathbf{H}=\mathbf{U}\left[\begin{array}{ll}
\mathbf{S} & 0 \\
0 & 0
\end{array}\right] \mathbf{V}^{T},
$$

where $\mathbf{U}$ and $\mathbf{V}$ are orthogonal matrices of $25 \times 25, \mathbf{S}=$ $\operatorname{diag}\left(\sigma_{1}, \sigma_{2}, \ldots, \sigma_{r}\right)(r \leq 25)$ and $r$ is the rank of the matrix $\mathbf{H}$ and the Jacobian matrix $\overline{\mathbf{J}}$. Therefore, the number of linearly related parameters is $25-r$. From (13) and (14), we can obtain

$$
\mathbf{V}^{T} \Delta \mathbf{X}=\left[\begin{array}{cc}
\mathbf{S}^{-1} & 0 \\
0 & 0
\end{array}\right] \mathbf{U}^{-1} \overline{\mathbf{J}}^{T} \Delta \overline{\mathbf{P}}
$$

Since $\mathbf{H}$ is a symmetric matrix, $\mathbf{V}^{T}=\mathbf{U}^{-1}$, and $\mathbf{V}$ are rotation matrices, then $\mathbf{V}^{T} \cdot \Delta \mathbf{X}$ is equivalent to $\Delta \mathbf{X}$. In this case, the linearly related structural parameters in $\Delta \mathbf{X}$ can be found by the elementary row transformation of the last 4 lines, as shown in

$$
\Delta \overline{\mathbf{P}}=\overline{\mathbf{J}} 1 \Delta \mathbf{X} 1,
$$

where $\overline{\mathbf{J}} 1$ is a matrix of $(3 n) \times r, \Delta \mathbf{X} 1$ is a vector of $r \times 1, \Delta \overline{\mathbf{P}}$ is the matrix of $(3 n) \times 1$, and $r$ is the rank of $\overline{\mathbf{J}} 1$. The calculation result shows that the rank of $\overline{\mathbf{J}} 1$ is 23 ; that is, $r=23$; thus there are two linearly related structural parameters. Specifically, we can obtain linearly related parameters by carrying out 


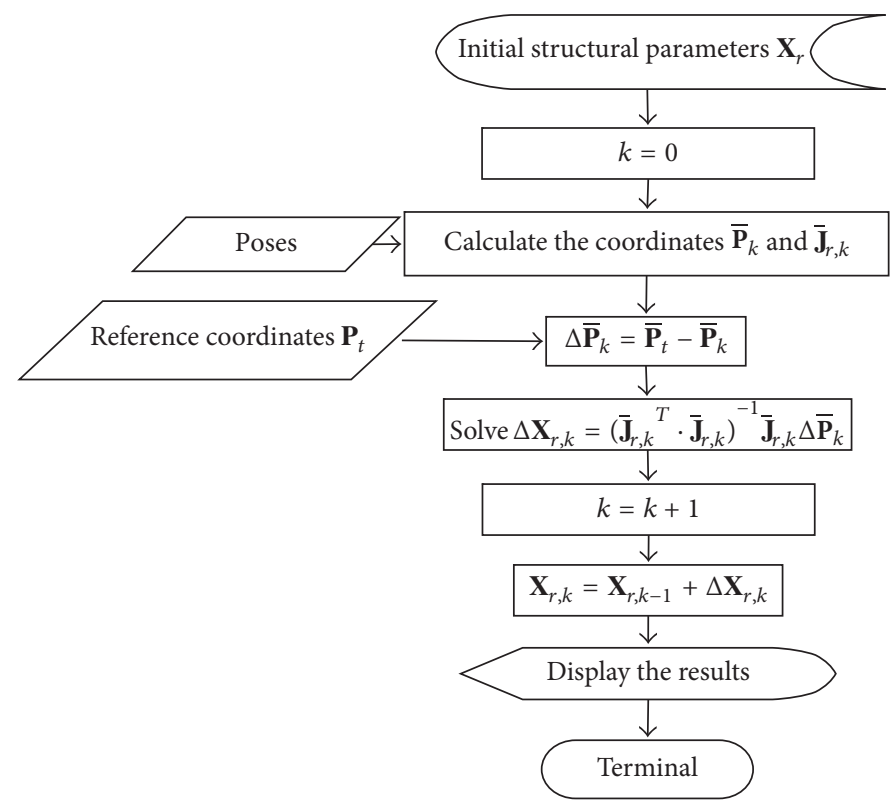

FIGURE 4: The flowchart of the identification procedure.

the singular value decomposition and the elementary row transform. As shown in (17), we find that $\Delta a_{6}$ is linearly related with $\Delta \theta_{6}$ and $\Delta d_{6}$ is linearly related with $\Delta \alpha_{6}$. Thus in the identification calculation, $\Delta \theta_{6}$ and $\Delta \alpha_{6}$ are treated as the redundant parameters and they do not need to be identified. Also, $\Delta a_{6}$ and $\Delta d_{6}$ can be determined as the redundant parameters and are not identified in the identification calculation, which can also reduce the dimension of the regressor matrices and thus the required calculation costs.

$$
\begin{aligned}
& \Delta a_{6}=l \Delta \theta_{6} \\
& \Delta d_{6}=l \Delta \alpha_{6} .
\end{aligned}
$$

3.4. Implementation of Identification Calculation. According to the analysis presented in last section, we know that there are two structural parameters which are related to others and do not need to be identified in terms of (12). Instead, they can be calculated based on (17) provided other 23 parameters are identified. Therefore, the two corresponding columns of the Jacobian matrix $\overline{\mathbf{J}} 1$ can be removed and the new identification equations can be obtained as shown in

$$
\Delta \mathbf{X}_{r}=\left(\overline{\mathbf{J}}_{r}^{T} \cdot \overline{\mathbf{J}}_{r}\right)^{-1} \overline{\mathbf{J}}_{r} \Delta \overline{\mathbf{P}}
$$

where $\Delta \mathbf{X}_{r}=\mathbf{X}_{r}(k)-\mathbf{X}_{r}(k-1)$ denotes the change between each iteration interval $[k-1, k]$, which is the vector of the structural parameters of the AACMM after the removal of $a_{6}$ and $d_{6}$, and $\overline{\mathbf{J}}_{r}$ is the Jacobian matrix after removal of the two columns of $a_{6}$ and $d_{6}$.

The overall identification algorithm has been carried out to solve (18) in Matlab, and the flowchart shown in Figure 4 provides the practical implementation of the suggested algorithm.
In the literature, there are many different identification methods, for example, PSO and GA. However, these algorithms need much more computational costs, which may limit their applicability for AACMM. The time consumed by the proposed identification calculation is relatively shorter than the widely used iteration identification methods such as PSO and GA; that is, we can get the results just after one time iteration calculation.

The step-by-step implementation procedure can be given as follows:

(1) initialize the structural parameters of $\mathbf{X}_{r, k}$ with the data in Table 1 and set iteration variable as $k=0$;

(2) calculate $\overline{\mathbf{P}}_{k}$ and $\overline{\mathbf{J}}_{r, k}$ with (2), (5), $\mathbf{X}_{r, k}$, and the poses acquired from the AACMM;

(3) calculate $\Delta \overline{\mathbf{P}}_{k}=\overline{\mathbf{P}}_{t}-\overline{\mathbf{P}}_{k}$ with the reference coordinates;

(4) calculate the least squares solution of $\Delta \mathbf{X}_{r, k}=\left(\overline{\mathbf{J}}_{r, k}{ }^{T}\right.$. $\left.\overline{\mathbf{J}}_{r, k}\right)^{-1} \overline{\mathbf{J}}_{r, k} \Delta \overline{\mathbf{P}}_{k}$

(5) set $k=k+1$ and correct the parameters as $\mathbf{X}_{r, k}=$ $\mathbf{X}_{r, k-1}+\Delta \mathbf{X}_{r, k}$ by using the composition $\Delta \mathbf{X}_{r, k}$.

\section{Experiments}

To show the effectiveness of the suggested modeling and identification algorithms, an experimental study was carried out based on an AACMM test rig, which is shown in Figure 5. The nominal point repeatability and volumetric accuracy of the AACMM are $0.05 \mathrm{~mm}$ and $0.06 \mathrm{~mm}$, respectively. However, the structural parameters and working principle of the AACMM are not clear for the users. This is the motivation for our current work, which is dedicated to estimate the parameters to achieve an identification model 


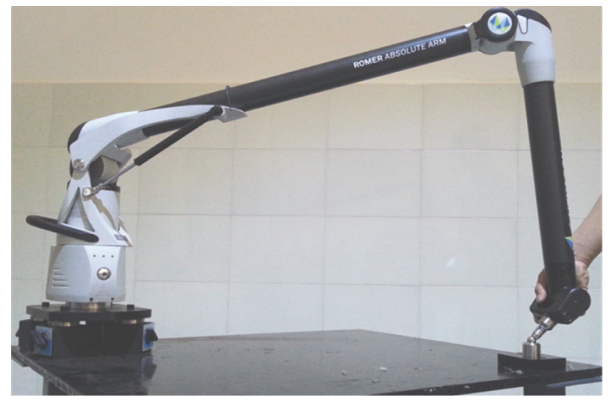

FIgURE 5: The articulated arm coordinate measuring machine.

TABLE 2: The movement uncertainties of AACMM before compensation.

\begin{tabular}{lcccc}
\hline Directions & Maximum $(\mathrm{max})(\mathrm{mm})$ & Standard deviation $(\mathrm{SD})(\mathrm{mm})$ & Average $($ ave $)(\mathrm{mm})$ & Absolute average $(\mathrm{AA})(\mathrm{mm})$ \\
\hline$x$ & 1.485 & 0.639 & -0.071 & 0.524 \\
$y$ & 1.366 & 0.478 & 0.439 & 0.545 \\
$z$ & -0.978 & 0.358 & -0.308 & 0.421 \\
\hline
\end{tabular}

of the AACMM. We conducted our experiments on the normal room temperature (in the range of $17^{\circ} \mathrm{C}$ to $23^{\circ} \mathrm{C}$ ) by a same operator. On the other hand, it should be noted that the main aim of this paper is to provide a parameter identification method for the AACMM and other similar link mechanisms. Thus, this paper does not consider the temperature compensation and calibration test according to ASME B.89.4.22, VDI/VDE 2617 Part 9 or ISO 10360 Part 12.

In the data acquisition procedure of the AACMM, many poses are used to obtain enough information about the AACMM. The information is very important for the following identification. And acquiring poses is commonly used in the calibration of AACMM and robots [15-17]. In this paper, we not only obtain many poses but also get the information of the structural parameters except measuring them directly. We operate the AACMM robot in extensive scenarios, and the joint angles and reference coordinates of 200 poses were acquired to test the uncertainty of the AACMM. The differences between the coordinates calculated joint angles based on the kinematic model and the reference coordinates are the movement uncertainty of the AACMM, which is shown in Figure 6. The maximum movement uncertainty is $1.485 \mathrm{~mm}, 1.366 \mathrm{~mm}$, and $-0.978 \mathrm{~mm}$ in the directions of $x, y$, and $z$, respectively, and the average of the absolute movement uncertainties are $0.524 \mathrm{~mm}, 0.545 \mathrm{~mm}$, and $0.421 \mathrm{~mm}$ for $x, y$, and $z$, respectively, as shown in Table 2 . Although the values of the structural parameters were estimated after repeated tests and measurements, the uncertainties of the AACMM were still relatively large. According to the analysis in Section 3, it is known that such movement uncertainty may be caused by the uncertainties of the structural parameters.

The significant uncertainties shown in Table 2 are unacceptable in practical application as this may severely deteriorate the overall measurement accuracy. Thus, the identification of uncertain structural parameters should be further conducted, which are then used to compensate the modeling errors in the $\mathrm{DH}$ model and then to improve the performance.
From the experimental studies, it is found that the movement uncertainty becomes relatively stable when the number of identification poses is greater than 30 , and it is almost invariant when the number of identification poses is larger than 50. Therefore, the joint angles and reference coordinates of 50 poses were acquired to identify the structural parameters of the AACMM, and the identification algorithm presented in Section 3.3 is used. The identification results are shown in Table 3. And the results can be reached just after one time iteration. Further experiments show that there is no further benefit to increase iteration times.

Compared to Table 1, one may find that the identified parameters in Table 3 are very close to the nominal values. This result indirectly validates the efficacy of the suggested identification algorithm. There are also some structural parameters (e.g., $d_{1}, d_{6}, \Delta \theta_{6}$, and $\alpha_{6}$ ) which are not changed for the reason that they are redundant and do not need to be identified. To further verify the identification results, another 200 groups of data were acquired to test the movement uncertainty of the AACMM. Figure 7 shows that the maximum movement uncertainties are $-0.112 \mathrm{~mm}, 0.116 \mathrm{~mm}$, and $0.117 \mathrm{~mm}$ in the directions of $x, y$, and $z$, respectively, and the averages of the absolute movement uncertainties are $0.036 \mathrm{~mm}, 0.038 \mathrm{~mm}$, and $0.044 \mathrm{~mm}$, which are summarized in Table 4. It is found from Tables 2 and 4 that the overall accuracy can be significantly improved by using the proposed identification and compensation method. The comparisons between the movement uncertainties after identification and the ones before identification are shown in Figure 8. As it is shown, all the movement uncertainty indexes are greatly improved after identification.

\section{Conclusions}

A constructive parameter identification approach for articulated arm coordinate measuring machines has been presented in this paper. A structural kinematic model is established 
TABLE 3: The identified structural parameters of AACMM.

\begin{tabular}{lcccc}
\hline Linkage number $i$ & $a_{i}[\mathrm{~mm}]$ & $d_{i}[\mathrm{~mm}]$ & $\Delta \theta_{i}\left[^{\circ}\right]$ & 0.087 \\
\hline 1 & -0.121 & 376.500 & 0.066 & -89.986 \\
2 & 61.942 & 0.016 & 0.001 & -90.026 \\
3 & 0.0324 & 750.658 & -0.021 & -89.997 \\
4 & 62.225 & -0.795 & 0.071 & -89.931 \\
5 & -0.036 & 500.287 & 0 & 90 \\
6 & 0.057 & 15 & 056 \\
\hline
\end{tabular}

$l=97.866 \mathrm{~mm}$.

TABLE 4: The movement uncertainty of AACMM after identification.

\begin{tabular}{lcccc}
\hline Directions & Maximum $(\mathrm{max})(\mathrm{mm})$ & Standard deviation $(\mathrm{SD})(\mathrm{mm})$ & Average $($ ave $)(\mathrm{mm})$ & Absolute average $($ AA) $(\mathrm{mm})$ \\
\hline$x$ & -0.112 & 0.044 & -0.003 & 0.036 \\
$y$ & 0.116 & 0.046 & 0.013 & 0.038 \\
$z$ & 0.117 & 0.053 & 0.007 & 0.044 \\
\hline
\end{tabular}

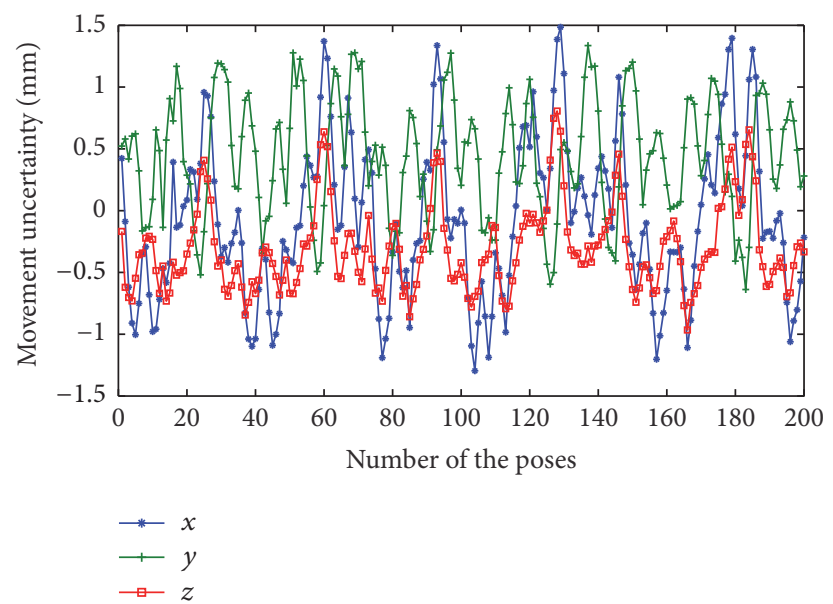

FIgURE 6: The movement uncertainties in the directions of $x, y$, and $z$ of AACMM without structural parameter identification and compensation.

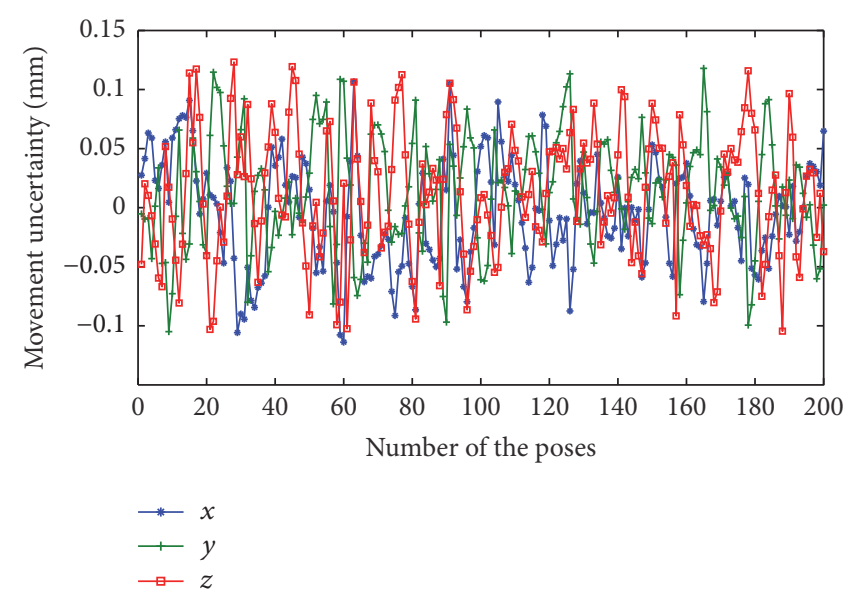

FIGURE 7: The movement uncertainties in the directions of $x, y$, and $z$ of AACMM with structural parameter identification and compensation. 


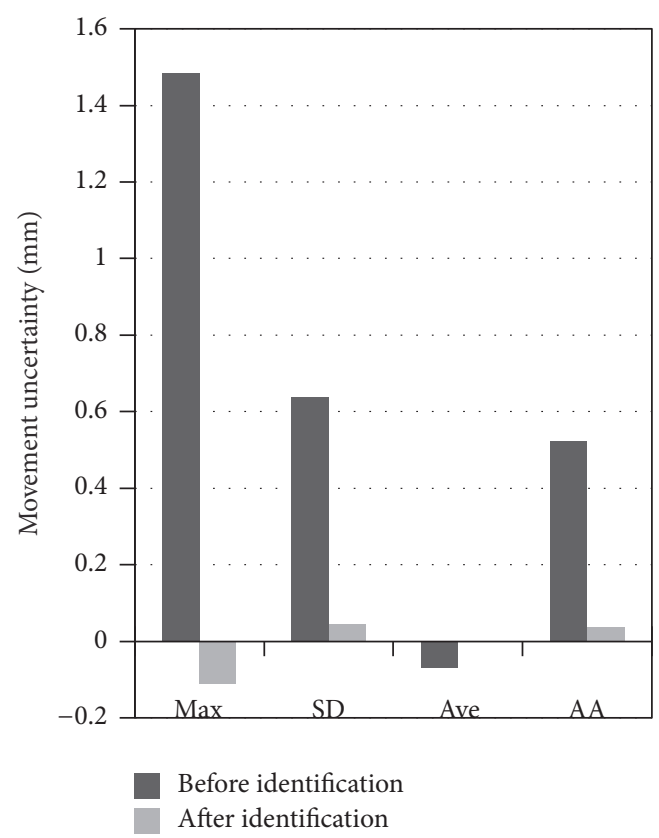

(a) In the direction of $x$

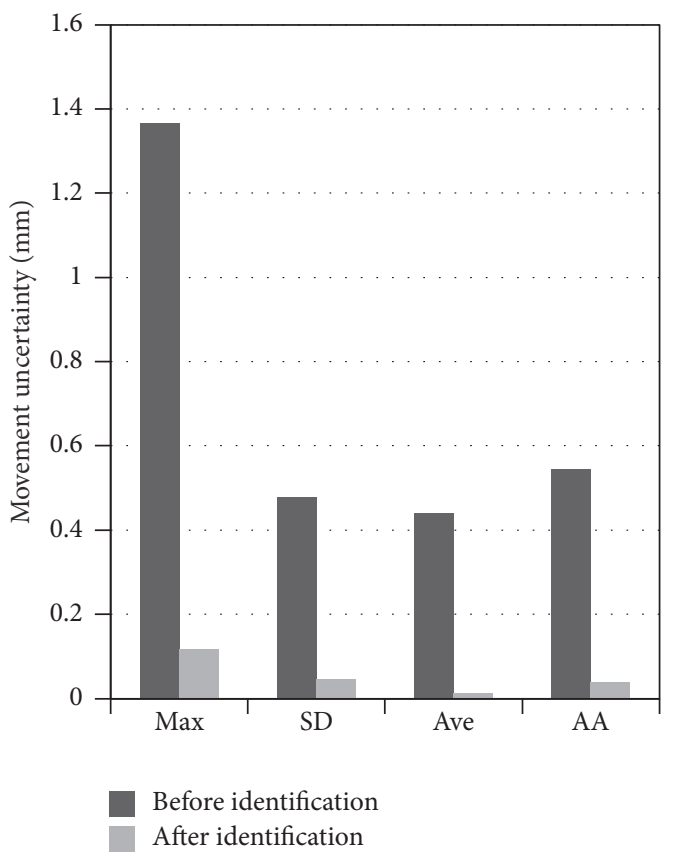

(b) In the direction of $y$

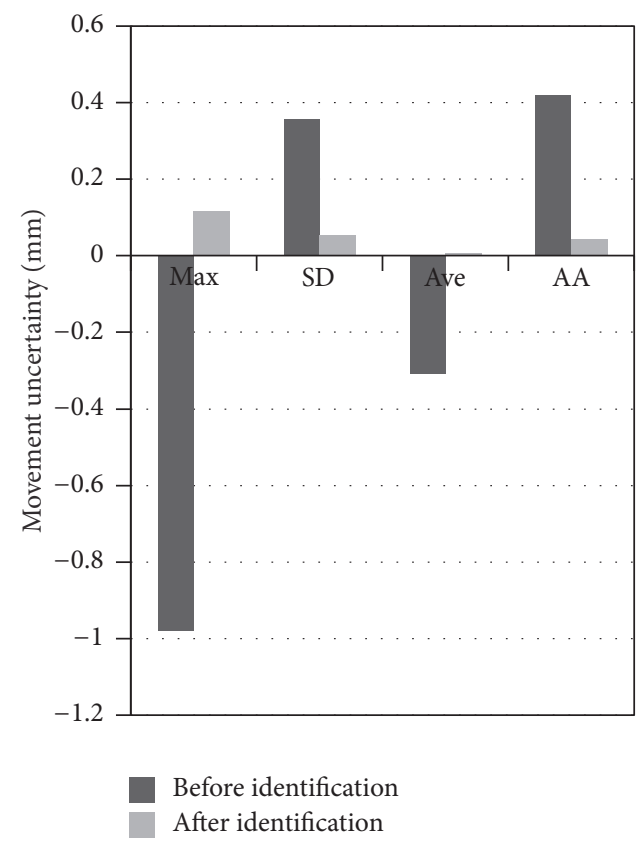

(c) In the direction of $z$

FIGURE 8: The comparison of the movement uncertainties in the directions of $x, y$, and $z$ of AACMM before and after identification.

based on the DH method and verified through experiments. Based on the difference between the coordinates of the probe calculated by the kinematic model and the reference coordinates, a mathematical parameter identification model is further developed to decrease the uncertainties in the $\mathrm{DH}$ model. The analysis of the Jacobian matrix in the identification model shows that there are two structural parameters which are related to others in our case studies. Therefore, these structural parameters are removed from the identification model. Then the structural parameter identification with the aim to get the least-square solution of the identification model can be carried out by using the obtained poses and the reference coordinates of the AACMM. To facilitate practical implementations, experimental studies have been 
conducted. These experimental results have revealed the effectiveness of the proposed structural parameter identification for AACMM.

The proposed modeling and identification method can be extended to the calibration of serial robots, where the identification method is required due to their dynamic operation environments. This will be further studied in our future work.

\section{Competing Interests}

The authors declare that they have no competing interests.

\section{Acknowledgments}

This work was supported by the National Natural Science Foundation of China (Grant no. 51465027).

\section{References}

[1] Y. Li and P. R. Nomula, "Surface-opening feature measurement using coordinate-measuring machines," International Journal of Advanced Manufacturing Technology, vol. 79, no. 9-12, pp. 19151929, 2015.

[2] W. Wang, G. Gao, Y. Wu, and Z. Chen, "Analysis and compensation of installation errors for circular grating angle sensors," Advanced Science Letters, vol. 4, no. 6-7, pp. 2446-2451, 2011.

[3] X. H. Li, B. Chen, and Z. R. Qiu, "The calibration and error compensation techniques for an Articulated Arm CMM with two parallel rotational axes," Measurement: Journal of the International Measurement Confederation, vol. 46, no. 1, pp. 603-609, 2013.

[4] D. Zheng, Z. Xiao, and X. Xia, "Multiple measurement models of articulated arm coordinate measuring machines," Chinese Journal of Mechanical Engineering (English Edition), vol. 28, no. 5, pp. 994-998, 2015.

[5] F. Romdhani, F. Hennebelle, M. Ge, P. Juillion, R. Coquet, and J. F. Fontaine, "Methodology for the assessment of measuring uncertainties of articulated arm coordinate measuring machines," Measurement Science and Technology, vol. 25, no. 12, Article ID 125008, 2014.

[6] A. Piratelli-Filho, F. H. T. Fernandes, and R. V. Arencibia, "Application of virtual spheres plate for AACMMs evaluation," Precision Engineering, vol. 36, no. 2, pp. 349-355, 2012.

[7] J. Sładek, K. Ostrowska, and A. Gaęska, "Modeling and identification of errors of coordinate measuring arms with the use of a metrological model," Measurement, vol. 46, no. 1, pp. 667-679, 2013.

[8] Y. Meng and H. Zhuang, "Autonomous robot calibration using vision technology," Robotics and Computer-Integrated Manufacturing, vol. 23, no. 4, pp. 436-446, 2007.

[9] J. U. Dolinsky, I. D. Jenkinson, and G. J. Colquhoun, "Application of genetic programming to the calibration of industrial robots," Computers in Industry, vol. 58, no. 3, pp. 255-264, 2007.

[10] I. Kovač and A. Frank, "Testing and calibration of coordinate measuring arms," Precision Engineering, vol. 25, no. 2, pp. 9099, 2001.

[11] J. Santolaria, J.-J. Aguilar, D. Guillomía, and C. Cajal, "A crenellated-target-based calibration method for laser triangulation sensors integration in articulated measurement arms,"
Robotics and Computer-Integrated Manufacturing, vol. 27, no. 2, pp. 282-291, 2011.

[12] J. Santolaria, A. C. Majarena, D. Samper, A. Brau, and J. Velázquez, "Articulated arm coordinate measuring machine calibration by laser tracker multilateration," The Scientific World Journal, vol. 2014, Article ID 681853, 11 pages, 2014.

[13] H. Hamana, M. Tominaga, M. Ozaki, and R. Furutani, "Calibration of articulated arm coordinate measuring machine considering measuring posture," International Journal of Automation Technology, vol. 5, no. 2, pp. 109-114, 2011.

[14] G. Gao, J. Lu, and H. Yang, "Study on the structrual parameter coupling of articulated arm coordinate measuring machines," Telkomnika, vol. 11, no. 5, pp. 2454-2459, 2013.

[15] L. Zhu, W. Li, Z. Pan, Y. Guo, and Q. Chen, "Research on parameter self-calibration method for partly-bonded articulated arm coordinate measuring machine," Chinese Journal of Scientific Instrument, vol. 35, no. 3, pp. 572-579, 2014.

[16] D. Zheng, Z. Xiao, and Y. Zhou, "Key technologies to improve articulated arm coordinate measuring machines' measurement accuracy," Journal of Hebei University of Science and Technology, vol. 35, no. 1, pp. 20-23, 2014.

[17] W. Cheng, Y. Fei, L. Yu, and R. Yang, "Probe parameters calibration for articulated arm coordinate measuring machine," in Proceedings of the 6th International Symposium on Precision Engineering Measurements and Instrumentation, vol. 7544 of Proceedings of SPIE, Hangzhou, China, August 2010.

[18] J. Denavit and R. S. Hartenberg, "A kinematic notation for lower-pair mechanisms based on matrices," ASME Journal of Applied Mechnics, vol. 22, no. 6, pp. 215-221, 1955.

[19] L. Q. Zhu, W. X. Li, Z. K. Pan, Y. K. Guo, and Q. S. Chen, "Coordinate measuring method with two operation modes based on the adjustable articulated arms," Optical Engineering, vol. 53, no. 12, Article ID 122407, 2014.

[20] A. Brau, M. Valenzuela, J. Santolaria, and J. J. Aguilar, "Evaluation of different probing systems used in articulated arm coordinate measuring machines," Metrology and Measurement Systems, vol. 21, no. 2, pp. 233-246, 2014.

[21] R. J. Lapeer, S. J. Jeffrey, J. T. Dao et al., "Using a passive coordinate measurement arm for motion tracking of a rigid endoscope for augmented-reality image-guided surgery," International Journal of Medical Robotics and Computer Assisted Surgery, vol. 10, no. 1, pp. 65-77, 2014.

[22] G.-M. Daniel, B. Joaquín, C. Eduardo, and M.-P. Susana, "Influence of human factor in the AACMM performance: a new evaluation methodology," International Journal of Precision Engineering and Manufacturing, vol. 15, no. 7, pp. 1283-1291, 2014.

[23] G. Gao, W. Wang, and J. Zhou, "Study on the error transfer of articulated arm coordinate measuring machines," TELKOMNIKA Indonesian Journal of Electrical Engineering, vol. 11, no. 2, pp. 637-641, 2013.

[24] S. Sagara and Y. Taira, "Cooperative manipulation of a floating object by some space robots: application of a tracking control method using the transpose of the generalized Jacobian matrix," Artificial Life and Robotics, vol. 12, no. 1-2, pp. 138-141, 2008.

[25] G. Pond and J. A. Carretero, "Formulating Jacobian matrices for the dexterity analysis of parallel manipulators," Mechanism and Machine Theory, vol. 41, no. 12, pp. 1505-1519, 2006. 


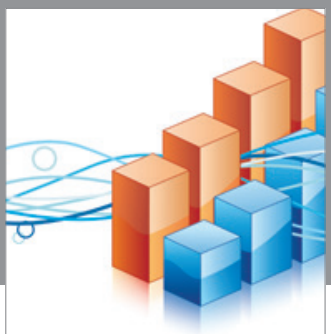

Advances in

Operations Research

vatem alat4

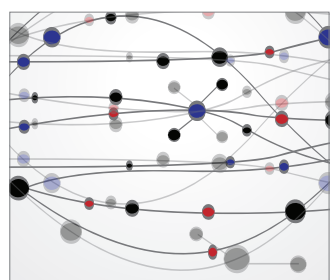

\section{The Scientific} World Journal
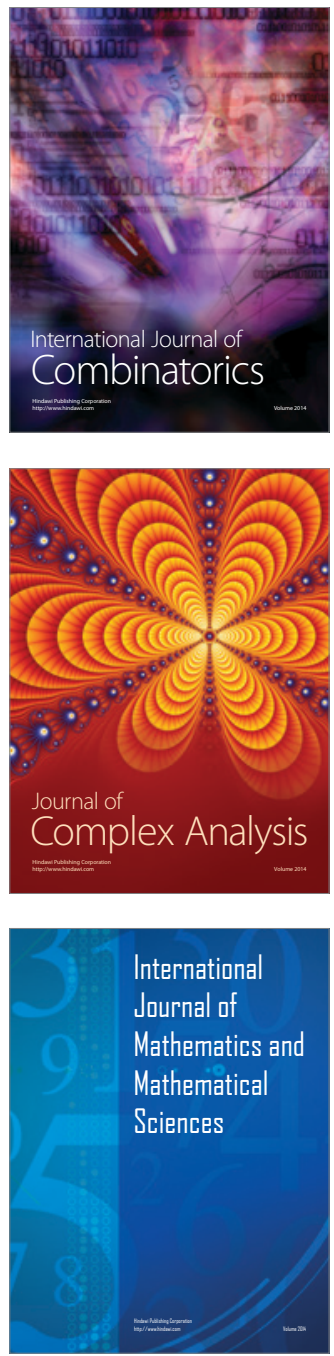
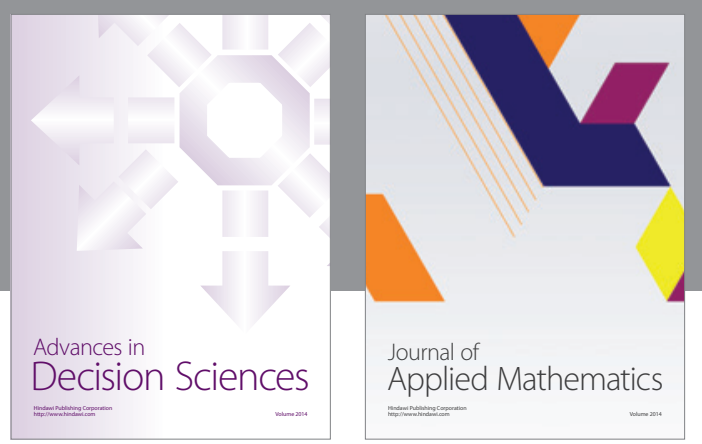

Algebra

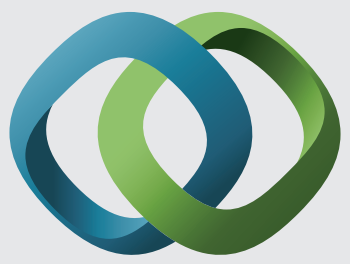

\section{Hindawi}

Submit your manuscripts at

http://www.hindawi.com
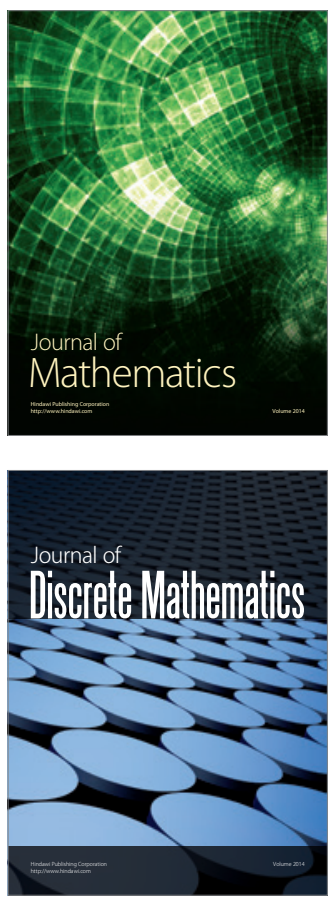

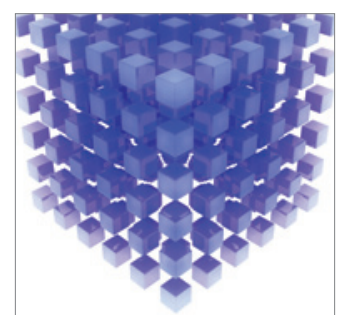

Mathematical Problems in Engineering
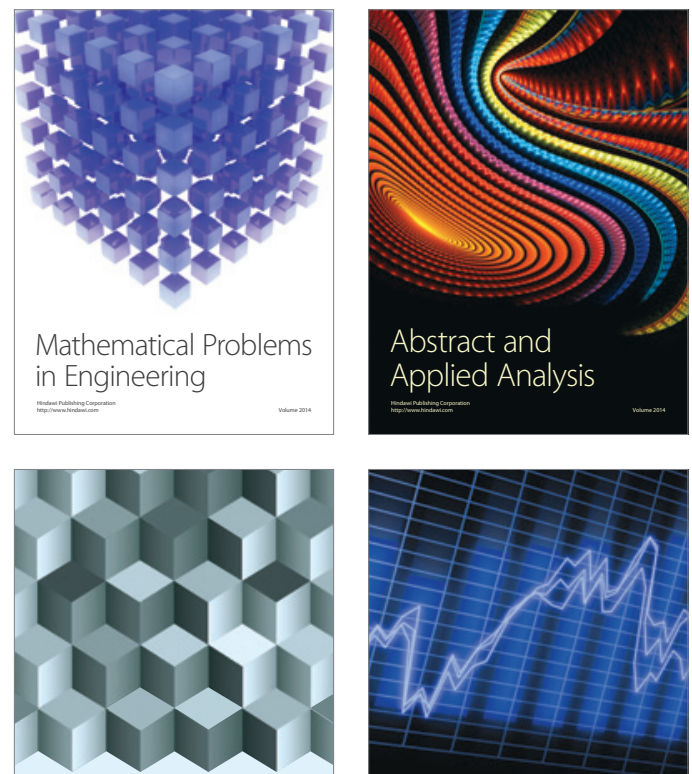

Journal of

Function Spaces

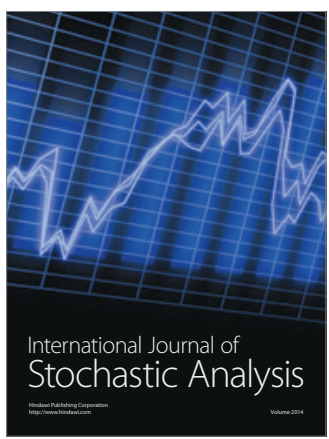

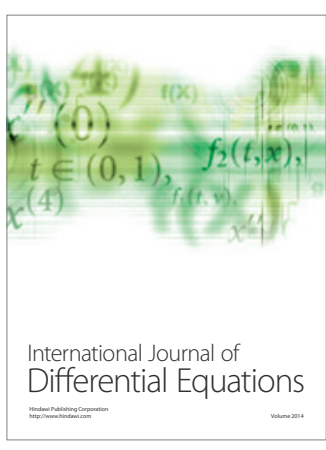
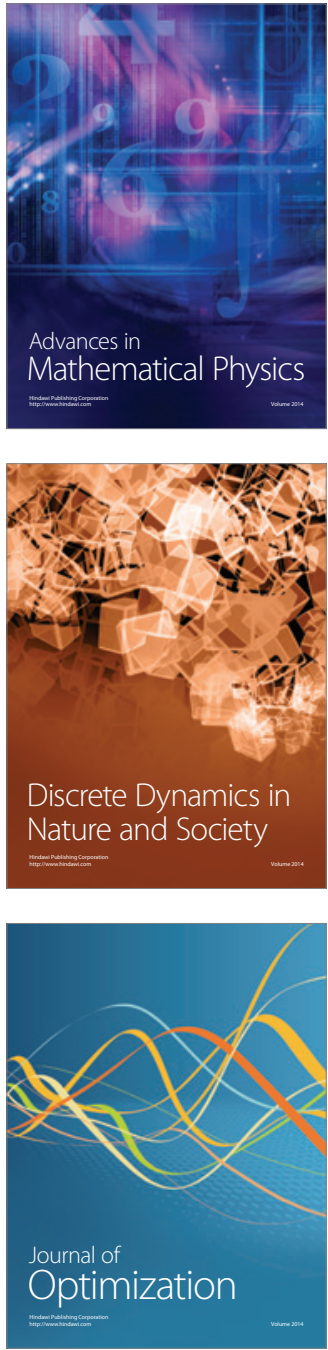